\title{
Photoconductively Loaded Plasmonic Nanoantenna as Building Block for Ultracompact Optical Switches
}

\author{
Nicolas Large, ${ }^{\dagger,}$ Martina Abb, ${ }^{\S}$ Javier Aizpurua, ${ }^{\dagger}$ and Otto L. Muskens ${ }^{*}, \S$ \\ ${ }^{\dagger}$ Centro de Fisica de Materiales CSIC-UPV/EHU and Donostia International Physics Center, DIPC, Paseo Manuel \\ Lardizabal 4, Donostia-San Sebastian 20018, Spain, ${ }^{*}$ CEMES/Université de Toulouse, France, and ${ }^{\S}$ School of Physics \\ and Astronomy, University of Southampton, Highfield, Southampton SO1 7 1BJ, United Kingdom
}

\begin{abstract}
We propose and explore theoretically a new concept of ultrafast optical switches based on nonlinear plasmonic nanoantennas. The antenna nanoswitch operates on the transition from the capacitive to conductive coupling regimes between two closely spaced metal nanorods. By filling the antenna gap with amorphous silicon, progressive antenna-gap loading is achieved due to variations in the free-carrier density in the semiconductor. Strong modification of the antenna response is observed both in the far-field response and in the local near-field intensity. The large modulation depth, low switching threshold, and potentially ultrafast time response of antenna switches holds promise for applications ranging from integrated nanophotonic circuits to quantum information devices.
\end{abstract}

KEYWORDS Plasmonics, nanoantennas, nanoparticles, ultrafast switches

$\mathrm{P}$ lasmonics has emerged recently as an extremely promising technological research area, owing to rapid advances in nanofabrication and modeling. ${ }^{1-3}$ Miniaturized nanoplasmonic devices hold the potential to become one of the key nanotechnologies capable of combining electric and photonic components on the same chip. ${ }^{2}$ Of special interest for nanoscale control over light are metal nanoantennas, capable of concentrating optical fields into a subwavelength volume. ${ }^{4-7}$ Analogous to their radiowave counterparts, nanoantennas support standing-wave electromagnetic resonances at visible and infrared wavelengths. Nanoantennas of various geometries have been applied successfully in nonlinear optics, ${ }^{4,5,8,9}$ nanoscale photodetectors, ${ }^{10}$ fluorescence enhancement, ${ }^{11-14}$ high-harmonic generation, ${ }^{15}$ and single-molecule detection. ${ }^{16}$

Active control over subwavelength optical fields is of importance for optical communication, sensing, and quantum information technology. In the terahertz range, the conductivity of semiconductors has been used recently to control the transport of terahertz waves using coupling to plasmonic modes on surfaces and nanostructures. ${ }^{17,18}$ Alloptical control over plasmons in the optical range has recently been demonstrated using planar metal films, ${ }^{20,21}$ hole arrays, ${ }^{22}$ and waveguided gold gratings. ${ }^{23}$ For single small metal nanoparticles, ultrafast heating, and coherent vibrations of the metal particle give rise to broadening and a spectral shift of its plasmon resonances. ${ }^{24-26}$ These effects

* To whom correspondence should be addressed, O.Muskens@soton.ac.uk. Received for review: 01/17/2010

Published on Web: 04/20/2010 are however not large enough for full optical control at the single-particle level for practical pump powers.

In this communication, we explore the functionality of plasmonic nanoantennas as novel building blocks for ultracompact nonlinear photonic devices. We propose that the small footprint, large light-matter interaction strength, and fast dynamics of single plasmonic nanoantennas can be used to design a new type of optical switch for controlling both the far-field and near-field distribution of light. Tunability of the antenna by impedance loading of its nanogap using a dielectric medium has recently been described theoretically ${ }^{27}$ and experimentally. ${ }^{28}$ In this work we explore a related but conceptually very distinct approach using photoconductive loading of the antenna gap. As many concepts in nanoplasmonics, photoconductive switching draws on analogies in the radiowave regime. ${ }^{29}$ The principle is based on the transition from capacitive to conductive coupling between two plasmon modes when bringing two nanoparticles into physical contact. ${ }^{30-32}$ Recently near-field investigations have shown control over progressive loading of a nanoantenna, which could be understood within the framework of circuit theory. ${ }^{33}$ We show here that photoexcited free carriers can be used to short circuit the antenna arms, leading to a strong modification of both the spectral resonance structure and near-field mode-profile. As the plasmonic antenna switch is based on a strong confinement of optical fields in space rather than in time, the antenna switch can operate at very low, picojoule, switching energies while potentially reaching a much faster, femtosecond, ${ }^{20,26}$ response than microphotonic switching devices. ${ }^{34,35}$

The response of cylindrical gold nanoantennas is calculated using the boundary element method (BEM) in a 


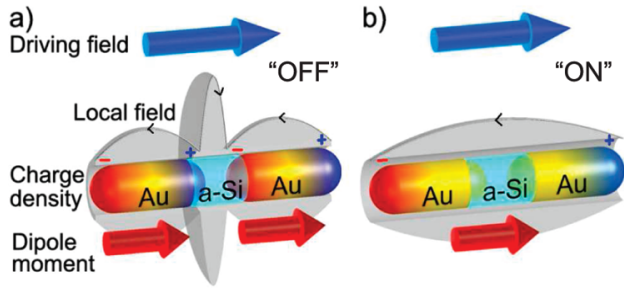

FIGURE 1. Illustration of the principle of antenna switching using a photoconductive gap, showing the fundamental mode of an unswitched ("OFF") (a) and a switched ("ON") (b) nanoantenna.

full electromagnetic calculation, thus including retardation. ${ }^{36,37}$ This method consists in solving Maxwell's equations by means of a distribution of surface charge densities and currents at the surfaces of the objects that interact self-consistently with the incoming field. The nanoantennas consist of two closely spaced cylindrical rods with their long axes aligned parallel as shown schematically in Figure 1. The rods are taken to have a hemispherical end-cap morphology. We consider gap antennas in absence of a substrate as this provides the simplest model system, to which additional elements like a dielectric substrate can be added. In experiments a suitable geometry can be chosen to decouple the substrate from the nanoantenna or match the surrounding dielectric. For the antenna switches the interparticle gap is loaded with amorphous silicon (a-Si). Amorphous silicon is chosen for its large electronic band gap of $1.6 \mathrm{eV}$, high free-carrier nonlinearity, and further for its wide application range and compatibility with many technological processes. ${ }^{38}$ The nonlinear optical response of crystalline silicon has been shown to be dominated by free carrier absorption, with a much weaker contribution from gap filling and band structure renormalization. ${ }^{39,40}$ As similar arguments hold for a-Si, we have calculated the dielectric function $\tilde{\epsilon}(\omega)$ of photoexcited a-Si by combining experimental dielectric function $\tilde{\epsilon}_{\text {exp }}$ taken from Aspnes et al. ${ }^{41}$ with the free-carrier Drude response, ${ }^{39}$ resulting in

$$
\tilde{\epsilon}(\omega)=\tilde{\epsilon}_{\exp }(\omega)-\left(\frac{\omega_{\mathrm{pl}}}{\omega}\right)^{2} \frac{1}{1+i \frac{1}{\omega \tau_{\mathrm{D}}}}
$$

where $\omega_{\mathrm{pl}}=\left(N_{\mathrm{eh}} e^{2} / \epsilon_{0} m_{\mathrm{opt}}{ }^{*} m_{\mathrm{e}}\right)^{1 / 2}$ denotes the plasma frequency, with $N_{\text {eh }}$ the free carrier density, $m_{\text {opt }}{ }^{*}=\left(m_{\mathrm{e}}{ }^{*-1}\right.$ $\left.+m_{\mathrm{h}}{ }^{*-1}\right)^{-1}$ the optical effective mass of the carriers, and $\tau_{\mathrm{D}} \sim 10^{-14} \mathrm{~s}$ the Drude relaxation time. The optical effective mass for a-Si, $m_{\text {opt }^{*}}{ }^{*}=0.17$, is estimated to be close to the value of crystalline silicon. ${ }^{39}$ The dielectric function $\tilde{\epsilon}(\omega)$ was calculated from eq 1 for values of the free-electron density $N_{\text {eh }}$ ranging from 0 to $10^{22} \mathrm{~cm}^{-3}$. Results are shown in Figure 2a, where we have plotted the real and imaginary parts of the refractive index $\tilde{\epsilon}^{1 / 2} \equiv$ $n+i \kappa$. For values of $N_{\text {eh }}$ above $10^{21} \mathrm{~cm}^{-3}$, a strong
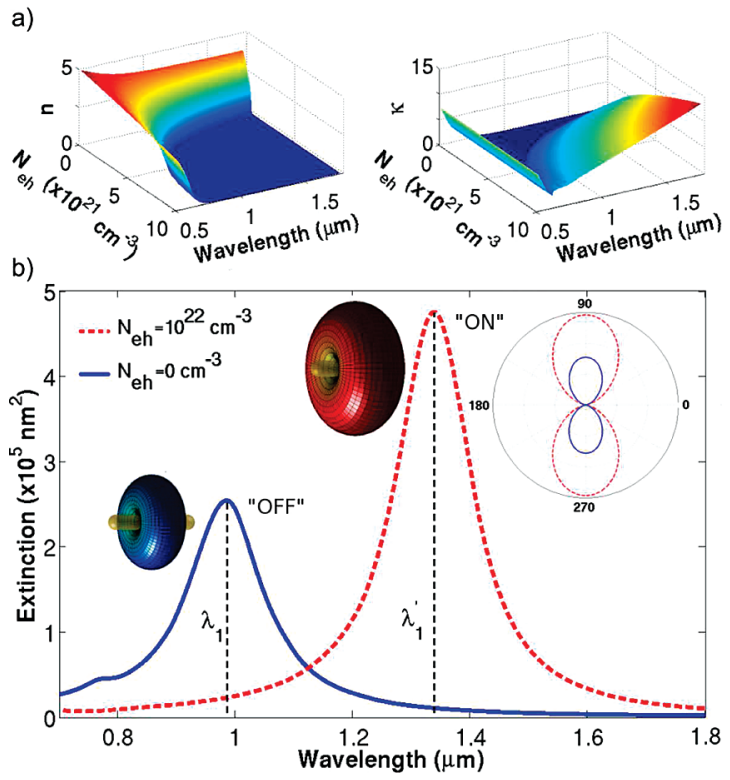

FIGURE 2. (a) Real (left panel) and imaginary (right panel) parts of the refractive index $\epsilon^{-1 / 2} \equiv n+i \kappa$, calculated using eq 1 as a function of the wavelength and the photoexcited free-carrier density $N_{\text {eh. }}$ (b) Optical extinction spectra for an $S=50 \mathrm{~nm}$ antenna, under unswitched (blue, "OFF") and switched (red dash, "ON") conditions, corresponding to respective carrier densities of $N_{\mathrm{eh}}=0 \mathrm{~cm}^{-3}$ and $10^{22} \mathrm{~cm}^{-3}$. The 3D radiation patterns are associated to the $\lambda_{1}$ and $\lambda_{1}{ }^{\prime}$ resonances of each switching mode. The inset represents the $2 \mathrm{D}$ cross section of these radiation patterns.

modification of the refractive index occurs corresponding to the formation of a free-carrier plasma. A critical density $N_{\text {eh }}{ }^{\text {crit }}$ can be defined as the transition from a primarily capacitive (dielectric) to primarily conductive (metallic) loading of the antenna gap, given by the condition $\kappa(\omega)>$ $n(\omega)$, or equivalently $\operatorname{Re}[\tilde{\epsilon}(\omega)]<0$. This condition yields an expression for the critical density

$$
N_{\mathrm{eh}}{ }^{\mathrm{crit}}(\omega)=\frac{\operatorname{Re}\left[\tilde{\epsilon}_{\mathrm{exp}}(\omega)\right] \varepsilon_{0} m_{\mathrm{opt}}{ }^{*}}{e^{2}}\left(\omega^{2}+1 / \tau_{\mathrm{D}}{ }^{2}\right)
$$

The critical threshold depends quadratically on the optical frequency $\omega$, while the influence of $\tau_{\mathrm{D}}$ becomes prominent for $\tau_{\mathrm{D}}<1 / \omega \sim 10^{-15} \mathrm{~s}$, where it results in an overall shift of $N_{\mathrm{eh}}{ }^{\text {crit }}$ to higher carrier densities.

The principle of operation of the nanoantenna switch is illustrated in Figure 1. In the unswitched case (denoted as "OFF"), the antenna supports half wavelength resonances over its individual arms. For gap sizes below $50 \mathrm{~nm}$, these half-wave modes are hybridized into a symmetric combination by the capacitive interaction between the two rods. ${ }^{42}$ Figure $1 \mathrm{~b}$ shows the response of the same antenna above the free-carrier switching threshold (denoted as "ON") given by eq 2 . As the antenna arms are conductively coupled, the antenna now supports a half-wave resonance over the full antenna length. As we will show below, the conductive gap 

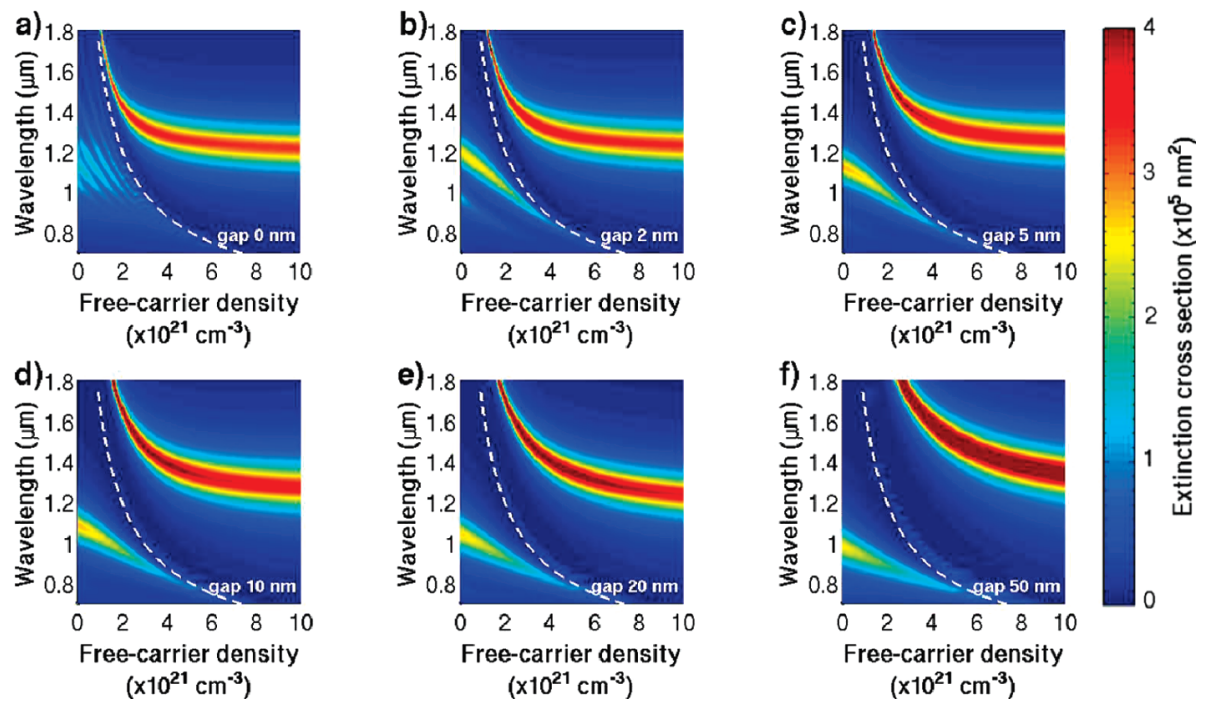

FIGURE 3. Color density maps of the antenna spectral response as a function of photoexcited free-carrier concentration $N_{\text {eh }}$, for antennas with gap sizes 0 (a), 2 (b), 5 (c), 10 (d), 20 (e), and $50 \mathrm{~nm}$ (f).

loading results in strong modifications of both the far-field antenna response and the near-field mode profiles.

Figure $2 \mathrm{~b}$ presents the effect of photoconductive switching on the far-field resonances of a nanoantenna with a gap width $S$ of $50 \mathrm{~nm}$. We present here the effect of a stationary carrier density; dynamic effects will be discussed further below. For this antenna, the capacitive interaction between the two nanorods is relatively weak and the resonances resemble those of the individual nanorods. Far-field extinction spectra were calculated for free carrier densities $N_{\text {eh }}$ below $\left(0 \mathrm{~cm}^{-3}\right.$, solid blue) and far above $\left(10^{22} \mathrm{~cm}^{-3}\right.$, dashed red) the switching threshold. The unswitched antenna shows a strong resonance at a wavelength $\lambda_{1}$ of $980 \mathrm{~nm}$, which can be attributed to the fundamental dipole modes of the uncoupled antenna arms. ${ }^{37}$ Photoconductive switching induces a shift of the resonance position from $\lambda_{1}$ to $\lambda_{1}{ }^{\prime}$ over $350 \mathrm{~nm}$, or a relative shift $\left(\lambda_{1}{ }^{\prime}-\lambda_{1}\right) / \lambda_{1}$ of $36 \%$. This switching effect is many times larger than that typically observed using dielectric loading. ${ }^{22,28}$ Importantly, the sharp resonance profile of a dipole antenna results in a large switching contrast of the extinction $\sigma_{\text {on }} / \sigma_{\text {off }}$ of 44 at the new resonance wavelength $\lambda_{1}{ }^{\prime}=1.33 \mu \mathrm{m}$ and inverse contrast $\sigma_{\text {off }} / \sigma_{\text {on }}$ of 11 at $\lambda_{1}=980 \mathrm{~nm}$. Calculated far-field radiation patterns corresponding to the two resonances of the unswitched and switched antenna are shown in the inset of Figure $2 \mathrm{~b}$. As both resonances correspond to a dipolar mode, no change is observed in the angular distribution pattern apart from an overall increase in radiative efficiency. This increase indicates that a larger antenna is formed under short-circuit conditions compared to the capacitive situation where there are two smaller antennas.

Figure 3 explores into more detail the antenna far-field response with progressive conductive loading for antennas with varying gap dimensions. We have calculated the optical extinction of nanoantennas with gap sizes $S$ ranging from 0 to $50 \mathrm{~nm}$. The dashed white lines in Figure 3 indicate the strongly wavelength dependent switching threshold for $N_{\text {eh }}{ }^{\text {crit }}$ for our model a-Si. The results of Figure $3 f$ correspond to the antenna of Figure $2 \mathrm{~b}(S=50 \mathrm{~nm})$. The general behavior of the resonance structure with increasing freecarrier density can be understood using basic circuit theory. ${ }^{27,33}$ The blue shift of the antenna resonance below the switching threshold, as the carrier density is increased, can be understood using a simple resistor model. One can think of this coupled-antenna resonance as a pure capacitive cavity where the positive and negative polarization charges act as a capacitor. As we increase now the carrier density in the bottleneck of the cavity, there is a reduction of the charges (reduction of the Coulomb interaction), and there will be a blue shift which is proportional to the reduction of the area of the capacitive coupling. There is a point where the current flow is so large that this capacitive mode at the cavity cannot be sustained any more; thus it gets completely damped and dies out.

The emergence of a new resonance at longer wavelengths above the critical switching threshold corresponds to the transition of a capacitively coupled to a conductively coupled cavity, where the strong dispersion results from the peculiar charge density pattern which piles up positive and negative charges at the end of the antenna. Starting from the purely conductive mode at $10^{22} \mathrm{~cm}^{-3}$, as the carrier density decreases and therefore the gap becomes less conductive, this mode would present net charge in one of the arms of the antenna and negative in the other. As this configuration is not physically possible, this modes shifts dramatically to longer wavelengths, and eventually damps and dies out. ${ }^{31,33}$ We point out that the spectral mode in the strongly dispersive regime is relatively narrow, which indicates a reduced damping of this mode. As a consequence of the strong wavelength dependence of $N_{\text {en }}{ }^{\text {crit }}$, the antenna can be simultaneously switched and unswitched in different parts of the spectrum. In this 

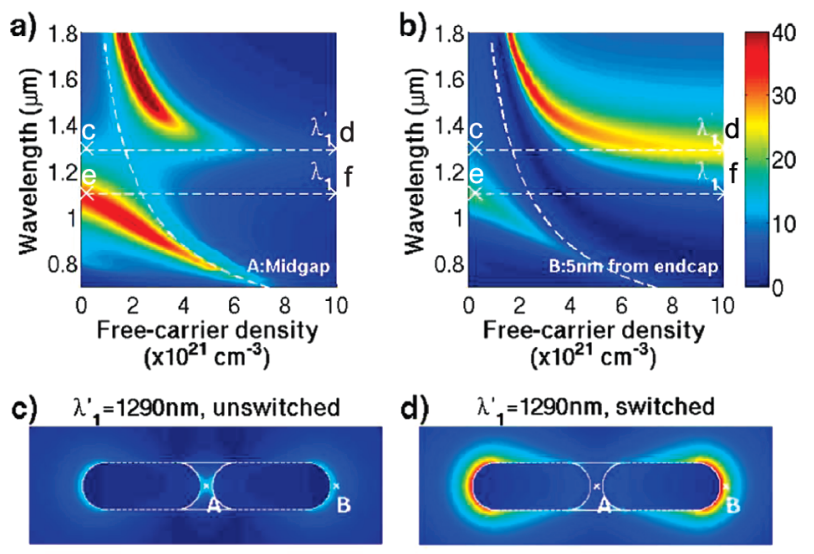

$\left(\mathrm{x} 10^{21} \mathrm{~cm}^{-3}\right)$

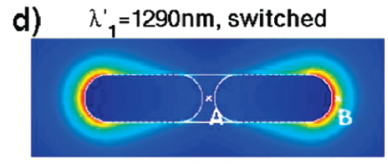

e) $\lambda_{1}=1100 \mathrm{~nm}$, unswitched
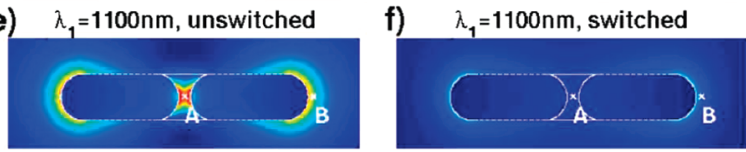

FIGURE 4. Near-field intensity maps calculated for a antenna of $L$ $=100 \mathrm{~nm}$ arm length and $S=10 \mathrm{~nm}$ gap size, as a function of wavelength and free-carrier density, for the antenna midgap (a) and $5 \mathrm{~nm}$ away from the antenna arms (b). (c-f) Near-field intensity maps around the antenna of (a) for resonance wavelength $\lambda_{1}{ }^{\prime}$ (c, d) and $\lambda_{1}(\mathrm{e}, \mathrm{f})$, under unswitched $\left(N_{\mathrm{eh}}=0 \mathrm{~cm}^{-3}\right)(\mathrm{c}, \mathrm{e})$ and switched $\left(N_{\mathrm{eh}}=10^{22} \mathrm{~cm}^{-3}\right)(\mathrm{d}, \mathrm{f})$ conditions.

transitional regime, the antenna supports both conductively coupled modes at longer wavelength and capacitive modes at shorter wavelengths, which could be of interest for device applications.

The $S=50 \mathrm{~nm}$ gap width in Figure $3 \mathrm{f}$ represents the virtually uncoupled regime, where capacitive interaction is very weak. For these wide-gap antennas, the far-field photoconductive switching effect is the strongest, first because of the absence of the red shift associated with capacitive coupling, and second because the total antenna length is the largest, leading to a longer wavelength fundamental mode. In comparison, for the $S=2 \mathrm{~nm}$ antenna of Figure $3 \mathrm{~b}$, switching from the capacitive to conductive state does not produce a marked wavelength shift. For the $S=0 \mathrm{~nm}$ antenna, the presence of a conductive singularity results in a strongly renormalized mode structure. ${ }^{31}$ Photoconductive antenna switching in this case strongly modifies the conductive area between the antenna arms, allowing a proper antenna mode to be formed. Similarly, for the photoconductive switch to operate according to Figure 3, the contact between the antenna and semiconductor needs to be conductive, as any small (nanometer) separation will act as a vacuum gap which will suppress the photoconductive switching effect.

Together with the switching of the far-field radiative properties, active manipulation of the local near-fields around plasmonic nanoantennas will be of importance for applications involving nonlinear optics and SERS, ${ }^{4}$ quantum emitters, ${ }^{16}$ and coherent control. ${ }^{43}$ We calculated the near-field intensity at various positions around a photoconductive antenna switch. Panels $\mathrm{a}$ and $\mathrm{b}$ of Figure 4 show the intensity at the center of the antenna gap and $5 \mathrm{~nm}$ from the antenna
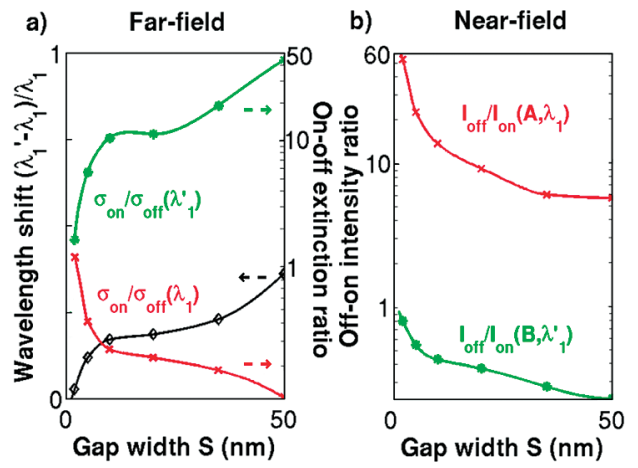

FIGURE 5. Figures of merit for switching operation for operation in far-field (a) and near-field (b). (a) Relative resonance shift $\left(\lambda_{1}{ }^{\prime}-\lambda_{1}\right)$ / $\lambda_{1}$ (left scale, linear) and on-off extinction ratio $\sigma_{\text {on }} / \sigma_{\text {off }}$ at wavelengths $\lambda_{1}$ and $\lambda_{1}^{\prime}$ (right scale, logarithmic), against antenna gap width $S$. (b) Near-field intensity off-on ratios $I_{\text {off }} / I_{\text {on }}$ at $\lambda_{1}$, point A (midgap) and $\lambda_{1}{ }^{\prime}$, point $\mathrm{B}$ (5 $\mathrm{nm}$ from tip).

ends for a dimer antenna with $S=10 \mathrm{~nm}$ gap width. This narrow-gap antenna supports a fundamental mode at a wavelength $\lambda_{1}$ of $1100 \mathrm{~nm}$ with a high local intensity enhancement of around 35 times the incident intensity in the gap region. The mode profile of this antenna resonance can be observed in detail in the near-field maps of Figure $4 \mathrm{c}-\mathrm{f}$. Photoconductive switching of the antenna results in a strong quenching of the midgap intensity by an order of magnitude, due to the redistribution of charges associated with the suppression of the gap capacitor (Figure 4e,f). A new mode is formed at a wavelength $\lambda_{1}{ }^{\prime}$ of $1290 \mathrm{~nm}$, where the intensity is mainly concentrated around the end points of the antenna and the midgap intensity is absent (Figure 4c,d). Remarkably, Figure 4 a shows that a transitional regime exists above $N_{\text {eh }}{ }^{\text {crit }}$ where the new mode shows a large midgap intensity, even exceeding that of the purely capacitive antenna. It should be noted that here the semiconductor still is partly dielectric, as otherwise the fields in the gap would be suppressed. The physical origin of this enhancement is again related to the particular charge distribution in the antenna in the crossover regime as discussed above.

For the rational design of antenna switches, we define figures of merit of antenna performance for far-field extinction and near-field intensity enhancements. For applications requiring large spectral shifts and extinction contrast, we calculate the relative resonance shift $\left(\lambda_{1}{ }^{\prime}-\lambda_{1}\right) / \lambda_{1}$ and the on-off extinction ratio $\sigma_{\text {on }} / \sigma_{\text {off }}$ at $\lambda_{1}$ and $\lambda_{1}{ }^{\prime}$. For the nearfield switching, key parameters of interest are the off-on ratios $I_{\text {off }} / I_{\text {on }}$ of the local intensity at the midgap A for the capacitive antenna resonance $\lambda_{1}$, and at the antenna end position $\mathrm{B}$ for the conductive antenna resonance $\lambda_{1}{ }^{\prime}$. Resulting values are shown in Figure 5 for the antennas with various gap sizes. The increasing capacitive loading for decreasing gap width $S$ drives the individual particle resonances $\lambda_{1}$ toward that of the half-wave antenna $\lambda_{1}{ }^{\prime}{ }^{4}$ resulting in a reduced far-field switching performance, i.e., values close to 1 . Therefore, for far-field switching, antennas with a large gap are favorable for achieving a large spectral shift 
and high switching contrast. We should bear in mind though that antennas with a large gap require more energy for switching and do not benefit as much from reduced switching thresholds at antenna resonances. For the near-field switching, antennas with a narrow gap are generally more favorable as these produce higher local field enhancements. However, the near field switching contrast depends strongly on the desired position and wavelength as observed in Figure 5b. Active manipulation of the local near-fields around plasmonic nanoantennas will be of importance for applications involving coherent control over local field amplitudes and phases ${ }^{43}$ and for active manipulation of quantum emitters. ${ }^{16}$

The free carrier densities required for nanoantenna switching at near-infrared wavelengths are higher than those in the terahertz range. ${ }^{17,18}$ However such densities are routinely achievable using ultrafast laser excitation of an electron-hole plasma. $^{39,40}$ In order to compare the switching energies required for photoconductive antenna switching with stateof-the-art microphotonic devices, we estimate the pumping energies for direct and two-photon optical excitation. In this estimate, we do not consider dynamical effects occurring on the time scale of the optical pump pulse, which will be discussed further below. For pulsed optical excitation on a time scale much shorter than carrier relaxation process in the system, the free carrier density $N_{\text {eh }}$ can be estimated from the incident optical fluence $F_{0}$ using ${ }^{38}$

$$
N_{\text {eh }}=\frac{F_{0}}{\hbar \omega}\left[\alpha_{0}+\frac{\beta F_{0}}{2 \sqrt{2 \pi} t_{0}}\right]
$$

where $\omega=2 \pi / \lambda, \alpha_{0}$ and $\beta$ are the linear and two-photon absorption coefficients, and $t$ is the time duration of the pulse. For excitation of a-Si in the telecommunication range using an ultrafast laser, a critical density $N_{\text {eh }}{ }^{\text {crit }}$ of around $10^{21}$ $\mathrm{cm}^{-3}$ is achieved via two-step absorption ( $\left.\beta \simeq 120 \mathrm{~cm} / \mathrm{GW}\right)$ at a fluence of $F_{0}=0.73 \mathrm{~mJ} / \mathrm{cm}^{2} .{ }^{38}$ Two-step absorption is an upconversion process where the electron is excited by two photons through an intermediate state, which in a-Si can be a midgap defect state. Similar switching fluences are obtained using linear absorption above the bandgap, where $\alpha_{0}>10^{5} \mathrm{~cm}^{-1}$. ${ }^{40}$ For excitation using a diffraction-limited spot of around $1 \mu \mathrm{m}^{2}$ area, the above fluence gives a switching energy of $7.3 \mathrm{pJ}$. This energy compares well to values achieved using microphotonic ring resonators (25 pJ) $)^{34}$ and photonic crystal nanocavities $(\sim 100 \mathrm{fJ}),{ }^{35}$ which are however intrinsically several orders of magnitude slower than plasmonic devices. ${ }^{20}$

The above switching energies are valid for ultrafast pulsed excitation. Under stationary (CW) pumping conditions, the carrier density will be limited by different relaxation mechanisms such as surface recombination, Auger processes, and electron diffusion. The ultrafast response of a-Si thin films has been extensively studied by Esser et al., ${ }^{40}$ who have shown that carrier densities in the $10^{21} \mathrm{~cm}^{-3}$ can be achieved using ultrafast laser absorption. Carrier trapping into localized states leads to a considerable reduction of the free carrier lifetime for a thin film compared to bulk crystalline Si. Above densities of $8 \times 10^{19} \mathrm{~cm}^{-3}$ another contribution to the relaxation time appears due to Auger processes involving spatially overlapping electron-hole pairs. The combined processes result in a relaxation rate of the order of $10^{13} \mathrm{~s}^{-1}$ at carrier densities around $10^{21} \mathrm{~cm}^{-3} .{ }^{40} \mathrm{An}$ additional limiting factor to the carrier concentration is carrier diffusion out of the gap region. For an $S=20 \mathrm{~nm}$ gap region and a carrier diffusion constant of $D_{\mathrm{e}} \simeq 40 \mathrm{~cm}^{2}$ / $\mathrm{S}$, electron diffusion will contribute to a relaxation rate of around $S^{2} / D \sim 10^{13} \mathrm{~s}^{-1}$, i.e., comparable to the Auger process.

In practice these relaxation rates balanced against the pumping rate will determine the stationary free carrier population that can be achieved. However they also provide the ultrafast time response of the nanoantenna switch desirable for many applications. Eventually all the energy deposited into the system will be converted into heat by electron-phonon relaxation on a time scale of picoseconds. ${ }^{26}$ In the extreme case that all this energy is dissipated entirely in the nanoantenna, it produces a temperature rise of around $30 \mathrm{~K}$. For this estimation we have used the lattice heat capacity of gold $C_{\mathrm{L}} \simeq 2.5 \times 10^{6} \mathrm{~J} / \mathrm{m}^{3} \mathrm{~K}$ and a total amount of $10^{6}$ generated electron-hole pairs per antenna and per pump pulse. This number of carriers follows from eq 3 for the switching fluence of $0.73 \mathrm{~mJ} / \mathrm{cm}^{2}$ and the antenna gap dimensions. Effects of overheating can be substantially reduced by embedding the antenna into an environment with a good thermal conductivity.

The combination of strong optical resonances with a high local field enhancement in the antenna gap opens up opportunities for optical pumping employing the mode structure and its dynamic modulation. Since only a nanometersized active volume has to be pumped, which is strategically located in the antenna gap, the estimated pump intensity required for switching can be significantly reduced through funneling of pump energy into the resonant antenna mode. Considering a typical 100 times resonant intensity enhancement in the antenna gap, ${ }^{4,37}$ we estimate an ultimate switching energy of around $100 \mathrm{fJ}$. In addition, it may be possible to employ the strong resonant enhancement of nonlinear optical phenomena in the feed gap, such as second harmonic and supercontinuum generation, ${ }^{4,5,9}$ to produce a nonlinear absorption complementary to two-step absorption. The above resonant reduction of the pumping threshold assumes that this energy can be deposited into the resonant mode before the switching itself changes the antenna mode structure. In the other limit of stationary resonant pumping, the dynamic switching of the antenna will result in optically bistable behavior. ${ }^{35}$ We propose that the nanoantenna switch can thus be used as a saturable absorber element. 
This application may hold substantial promise for semiconductor lasers, which currently operate in the sub-100-fs pulsed regime. ${ }^{44}$ The exact conditions for successful operation of plasmonic saturable absorbers will require extensive modeling which goes beyond our current work.

In conclusion, we have investigated a new class of nanoscale optical switches consisting of a plasmonic nanoantenna loaded with a photoconductive semiconductor material. By short circuiting the antennagap using a semiconductor free-carrier plasma, we have shown that the mode spectrum of the antenna shifts dramatically using only a very modest pumping energy. The nanoantenna switch can be used to control both far-field and near-field properties. In addition, we have identified a regime of partially conductive gap where a new plasmon mode is formed, which combines a narrow spectral profile with a large local-field enhancement in the antenna gap. The nanoantenna switch combines large modulation depth, low switching threshold, and potentially ultrafast time response. Although the switching threshold is relatively high compared to terahertz photoconductive devices, we expect that these carrier densities can be achieved using ultrafast optical pumping. The theoretical concept presented here is generally applicable to a wide range of experimental designs, which may involve top-down routes based on nanolithography ${ }^{4}$ or bottom-up colloidal or electrochemical approaches. ${ }^{45}$ Further optimization may be possible by considering antenna designs different from the coupled-dipole configuration. Nanoantenna switches open up new avenues to applications ranging from integrated photonics and ultrafast lasers to quantum information devices.

Acknowledgment. O.L.M. acknowledges support from EPSRC through Grant EP/H019669/1 and from HEFCE through a lectureship funded by SEPnet. M.A. is supported by HEFCE though a PhD studentship funded by SEPnet. J.A. acknowledges funding from the ETORTEK project inanoGUNE from the Basque Government and project FIS200766711-C01-01 of the Spanish Ministry of Innovation and Science.

\section{REFERENCES AND NOTES}

(1) Barnes, W. L.; Dereux, A.; Ebbesen, T. W. Nature 2003, 424, $824-$ 826.

(2) Ozbay, E. Science 2006, 311, 189.

(3) Maier, S. A., Plasmonics: fundamentals and applications, 1st ed.; Springer: New York, 2007.

(4) Mühlschlegel, P.; Eisler, H.-J.; Martin, O. J. F.; Hecht, B.; Pohl, D. W. Science 2005, 308, 1607.

(5) Schuck, P. J.; Fromm, D. P.; Sundaramurthy, A.; Kino, G. S.; Moerner, W. E. Phys. Rev. Lett. 2005, 94, No. 017402.

(6) Yu, N.; Cubukcu, E.; Diehl, L.; Bour, D.; Corzine, S.; Zhu, J.; Höfler, G.; Crozier, K. B.; Capasso, F. Opt. Express 2007, 15, $13272-$ 13281

(7) Bharadwaj, P.; Deutsch, B.; Novotny, L. Adv. Opt. Photonics 2009, 1,438 .

(8) Ghenuche, P.; Cherukulappurath, S.; Taminiau, T. H.; van Hulst, N. F.; Quidant, R. Phys. Rev. Lett. 2008, 101, 116805.

(9) Danckwerts, M.; Novotny, L. Phys. Rev. Lett. 2007, 98, No. 026104
(10) Tang, L.; Kocabas, S. E.; Latif, S.; Okyay, A. K.; Ly-Gagnon, D.-S.; Saraswat, K. C.; Miller, D. A. B. Nat. Photonics 2008, 2, 226.

(11) Farahani, J. N.; Pohl, D. W.; Eisler, H.-J.; Hecht, B. Phys. Rev. Lett. 2005, 95, No. 017402 .

(12) Kühn, S.; Hakanson, U.; Rogobete, L.; Sandoghdar, V. Phys. Rev. Lett. 2006, 97, No. 017402

(13) Muskens, O. L.; Giannini, V.; Sánchez Gil, J. A.; Gómez Rivas, J. Nano Lett. 2007, 7, 2871.

(14) Zoriniants, G.; Barnes, W. L. New J. Phys. 2008, 10, 105002.

(15) Kim, S.; Jin, J.; Kim, Y.-J.; Park, I.-Y.; Kim, Y.; Kim, S.-W. Nature 2008, 453, 757.

(16) Akimov, A. V.; Mukherjee, A.; Yu, C. L.; Chang, D. E.; Zibrov, A. S.; Hemmer, P. R.; Park, H.; Lukin, M. D. Nature 2007, 450, 402.

(17) Hendry, E.; Lockyear, M. J.; Gómez Rivas, J.; Kuipers, L.; Bonn, M. Phys. Rev. B 2007, 75, 235305.

(18) Chen, H.-T.; Padilla, W. J.; Cich, M. J.; Azad, A. K.; Averitt, R. D.; Taylor, A. J. Nat. Photonics 2009, 3, 148.

(19) Williams, C. R.; Andrews, S. R.; Maier, S. A.; FernándezDomínguez, A. I.; Martín-Moreno, L.; Garcia-Vidal, F. J. Nat. Photonics 2008, 2, 175.

(20) MacDonald, K. F.; Sámson, Z. L.; Stockman, M. I.; Zheludev, N. I. Nat. Photonics 2008, 3, 55 .

(21) Pacifici, D.; Lezec, H. J.; Atwater, H. A. Nat. Photonics 2007, 1, 402.

(22) Wurtz, G. A.; Pollard, R.; Zayats, A. V. Phys. Rev. Lett. 2006, 97 , No. 057402

(23) Zhang, X.; Sun, B.; Hodgkiss, J. M.; Friend, R. H. Adv. Mater. 2008, $20,4455$.

(24) van Dijk, M. A.; Lippitz, M.; Orrit, M. Phys. Rev. Lett. 2005, 95, 267406

(25) Park, S.; Pelton, M.; Liu, M.; Guyot-Sionnest, P.; Scherer, N. F. J. Phys. Chem. C 2007, 111, 116-123.

(26) Muskens, O. L.; Del Fatti, N.; Vallee, F. Nano Lett. 2006, 6, 552-556.

(27) Alù, A.; Engheta, N. Nat. Photonics 2008, 2, 307.

(28) Berthelot, J.; Bouhelier, A.; Huang, C.; Margueritat, J.; Colas-desFrancs, G.; Finot, E.; Weeber, J.-C.; Dereux, A.; Kostcheev, S.; Ibn El Ahrach, H.; Baudrion, A.-L.; Plain, J.; Bachelot, R.; Royer, P.; Wiederrecht, G. P. Nano Lett. 2009, 9, 3914.

(29) Panagamuwa, C. J.; Chauraya, A.; Vardaxoglou, J. C. IEEE Trans. Antennas Propag. 2006, 54, 449.

(30) Atay, T.; Song, J.-H.; Nurmikko, A. V. Nano Lett. 2004, 4, 1627.

(31) Romero, I.; Aizpurua, J.; Bryant, G. W.; García de Abajo, F. J. Opt. Express 2006, 14, 9988

(32) Lassiter, J. B.; Aizpurua, J.; Hernandez, L. I.; Brandl, D. W.; Romero, I.; Lal, S.; Hafner, J. H.; Nordlander, P.; Halas, N. J. Nano Lett. 2008, 8, 1212 .

(33) Schnell, M.; García-Etxarri, A.; Huber, A. J.; Crozier, K.; Aizpurua, J.; Hillenbrand, R. Nat. Photonics 2009, 3, 287.

(34) Almeida, V. R.; Barrios, C. A.; Panepucci, R. R.; Lipson, M. Nature 2004, 431, 1081 .

(35) Tanabe, T.; Notomi, M.; Mitsugi, S.; Shinya, A.; Kuramochi, E. Opt. Lett. 2005, 30, 2575.

(36) García de Abajo, F. J.; Howie, A. Phys. Rev. Lett. 1998, 80, 5180.

(37) Aizpurua, J.; Bryant, G. W.; Richter, L. J.; García de Abajo, F. J.; Kelley, B. K.; Mallouk, T. Phys. Rev. B 2005, 71, 235420.

(38) Ikeda, K.; Shen, Y.; Fainman, Y. Opt. Express 2007, 15, 17761.

(39) Sokolowski-Tinten, K.; von der Linde, D. Phys. Rev. B 2000, 61, 2643.

(40) Esser, A.; Seibert, K.; Kurz, H.; Parsons, G. N.; Wang, C.; Davidson, B. N.; Lucovsky, G.; Nemanich, R. J. Phys. Rev. B 1990, 41, 2879.

(41) Aspnes, D. E.; Studna, A. A.; Kinsbron, E. Phys. Rev. B 1984, 29, 768.

(42) Prodan, E.; Radloff, C.; Halas, N. J.; Nordlander, P. Science 2003, 302, 419 .

(43) Aeschlimann, M.; Bauer, M.; Bayer, D.; Brixner, T.; García de Abajo, F. J.; Pfeiffer, W.; Rohmer, M.; Spindler, C.; Steeb, F. Nature 2007, 446, 301.

(44) Quarterman, A. H.; Wilcox, K. G.; Apostolopoulos, V.; Mihoubi, Z.; Elsmere, S. P.; Farrer, I.; Ritchie, D. A.; Tropper, A. Nat. Photonics 2009, 3, 729 .

(45) Pen, D. J.; Mbindyo, J. K. N.; Carado, A. J.; Mallouk, T. E.; Keating, C. D.; Razavi, B.; Mayer, T. S. J. Phys. Chem. B 2002, 106, 74587462 . 